\title{
ON THE CONTINUOUS FUNCTION SPACE OF A BASICALLY DISCONNECTED SPACE ${ }^{1}$
}

\author{
DWIGHT B. GOODNER
}

Throughout this note we shall let $H$ be a Hausdorff space and let $C(H)$ be the space of bounded continuous real-valued functions on $H, C(H)$ having the usual supremum norm. Certain results (cf. e.g. [7]) suggest the possibility of showing that if a normed linear space $X$ is complemented in every superspace (cf. [1, pp. 94 and 120]), then $X$ is isomorphic to some space $C(H)$ over a Stone space $H$, and that if $X$ is isometric to some $C(H)$, then $H$ is basically disconnected. The purpose of this note is to extend a result of Dean [2, p. 391] for $C(H)$ where $H$ is extremally disconnected and compact to the case where $H$ is basically disconnected and normal. Our proof rests on an extension of James' technique [6, p. 900] for embedding the space $(m)$ of bounded sequences in $C(H)$ where $H$ is infinite, extremally disconnected and compact.

Let $r$ be a real number. We shall say that $H$ is basically disconnected if and only if the closure of every open set of the form

$$
G(f, r)=\{h: f(h)<r, h \in H, f \in C(H)\}
$$

is open. We note that an extremally disconnected space is basically disconnected, that a basically disconnected space is totally disconnected, and that in a normal space an open set is an $F_{\sigma}$ set if and only if it is a set of the form $G(f, r)$ (cf. [3, p. 15]).

Our first lemma contains a result of Dean [2, p. 391].

Lemma 1. If $H$ is an infinite basically disconnected normal Hausdorff space, if $W$ is an infinite open and closed subset of $H$, and if $h^{\prime}$ is a point in $W$, then $W-\left\{h^{\prime}\right\}$ contains an infinite open and closed set.

Proof. Suppose $W-N$ is finite whenever $N \subset W$ is a neighborhood of $h^{\prime}$. Then each point $h \neq h^{\prime}$ in $W$ is open. Hence each countably infinite subset $H^{\prime}$ of $W-\left\{h^{\prime}\right\}$ is an open $F_{\sigma}$ set and its closure $\bar{H}^{\prime}=H^{\prime} \cup\left\{h^{\prime}\right\}$ is open. It follows that if $H^{\prime}$ and $H^{\prime \prime}$ are countably infinite subsets of $W-\left\{h^{\prime}\right\}$, then $\bar{H}^{\prime} \cap \bar{H}^{\prime \prime} \supset\left\{h^{\prime}\right\} \neq \varnothing$ even though $H^{\prime}$ and $H^{\prime \prime}$ may be disjoint. But this is impossible because in a basically disconnected normal Hausdorff space, disjoint open $F_{\sigma}$ sets

Presented to the Society, November 17, 1962; received by the editors July 9, 1963 and, in revised form, July 9, 1964.

${ }^{1}$ Research supported in part by a grant from the Florida State University Research Council. 
have disjoint closures. Hence there is a neighborhood $N^{\prime}$ of $h^{\prime}$ such that $N^{\prime} \subset W$ and $W-N^{\prime}$ is infinite. If $f \in C(H)$ takes the value 0 on $W-N^{\prime}$, 1 at $h^{\prime}$, and 1 on $H-W$, the closure of $\left\{h: f(h)<\frac{1}{2}\right\}$ is an infinite open and closed subset of $W-\left\{h^{\prime}\right\}$.

Lemma 2. If $H$ is an infinite basically disconnected normal Hausdorff space, then $H$ contains an infinite sequence $\left\{V_{i}\right\}$ of pairwise disjoint, nonempty, open and closed sets. If $V$ is the closure of $U_{i=1}^{\infty} V_{i}$, then $V$ is open and closed.

Proof. We will construct the sequence inductively. Let $h_{1}$ be a point in $H$. By Lemma 1 there is an infinite open and closed subset $N_{1} \subset H-\left\{h_{1}\right\}$. Let $V_{1}=H-N_{1}$. We note that $h_{1} \in V_{1}$ and $V_{1}$ is open and closed. Suppose we have chosen pairwise disjoint open and closed sets $V_{1}, V_{2}, \cdots, V_{k}$ so that $h_{i} \in V_{i}$ and $N_{k}=H-\bigcup_{i=t}^{k} V_{i}$ is an infinite open and closed subset of $H$. Let $h_{k+1}$ be any point in $N_{k}$. Then by Lemma 1 there is an infinite open and closed set $N_{k+1} \subset N_{k}-\left\{h_{k+1}\right\}$. Let $V_{k+1}=N_{k}-N_{k+1}$. Then $h_{k+1} \in V_{k+1}$ and $V_{k+1}$ is open and closed. This completes the inductive construction.

Since each $V_{i}$ is an open $F_{\sigma}$ set, $\bigcup_{i=1}^{\infty} V_{i}$ is an open $F_{\sigma}$ set and it follows that $V$ is open and closed. This completes the proof.

James $[6$, p. 900$]$ embedded the space $(m)$ of bounded sequences in $C(H), H$ an infinite extremally disconnected compact Hausdorff space, by using an infinite sequence of pairwise disjoint open and closed subsets of $H$. Using Lemma 2 and James' procedure, we may embed $(m)$ in $C(H)$ where $H$ is an infinite basically disconnected normal Hausdorff space (cf. [5, p. 257]). If $\left\{h_{i}\right\}_{i=1}^{\infty},\left\{V_{i}\right\}_{i=1}^{\infty}$ and $V$ are as in Lemma 2, a suitable embedding, $Q$, may be defined by $Q(x)=f$ implies

$$
f(h)=\left\{\begin{array}{cl}
0 & \text { if } h \in H-V, \\
x(i) & \text { if } h \in V_{i},
\end{array}\right.
$$

where $x \in(m)$ and $f \in C(H)$.

Our theorem contains a result of Dean [2, p. 391].

THEOREM. Let $H$ be an infinite basically disconnected normal Hausdorff space and let the space $(m)$ of bounded sequences be embedded in $C(H)$ as above; that is, let $Q((m))=\left(m^{\prime}\right) \subset C(H)$. Then a subspace $B$ of $C(H)$ complementary to $\left(m^{\prime}\right)$ is isomorphic to $C(H)$ or is finite dimensional.

Proof. Let $f \in C(H)$. Define $T f$ to be the element of $\left(m^{\prime}\right)$ for which $T f(h)=f\left(h_{i}\right)$ for $h$ and $h_{i}$ in $V_{i}$ as in Lemma 2 . Then $T$ is a projection of $C(H)$ onto $\left(m^{\prime}\right)$, and $C(H)$ is the direct sum of $\left(m^{\prime}\right)$ and the null 
space $Y$ of $T$; that is, $C(H)=Y \oplus\left(m^{\prime}\right)$ (cf. [4, p. 91], [8, p. 538]). If the set $H^{\prime}$ of points in $H$ and not in the closure of $\bigcup_{i=1}^{\infty}\left\{h_{i}\right\}$ is finite, then $Y$ is finite dimensional (cf. [2, p. 392]).

If $H^{\prime}$ is infinite, then $H^{\prime}$ contains an infinite open and closed subset $H^{\prime \prime}$. For suppose each $V_{i}$ is finite. Then each $h_{i}$ is open and $\bigcup_{i=1}^{\infty}\left\{h_{i}\right\}$ is an open $F_{\sigma}$ set. It follows that the closure of $\bigcup_{i=1}^{\infty}\left\{h_{i}\right\}$ is open and, hence, that $H^{\prime}$ itself is an infinite open and closed set. Alternatively, suppose some $V_{i}$ is infinite. Then, by Lemma $1, V_{i}-\left\{h_{i}\right\}$ contains an infinite open and closed subset $H^{\prime \prime}$. In either case, by Lemma $2, H^{\prime \prime}$ contains an infinite sequence $\left\{V_{i}^{\prime}\right\}_{i=1}^{\infty}$ of nonempty, pairwise disjoint, open and closed subsets. Let $\left(m^{\prime \prime}\right)$ be the embedding of $(m)$ in $C(H)$ determined by the sequence $\left\{V_{i}^{\prime}\right\}_{i=1}^{\infty}$. Then $\left(m^{\prime \prime}\right)$ is a subspace of $Y=Z \oplus\left(m^{\prime \prime}\right)$ and $C(H)=Z \oplus\left(m^{\prime}\right) \oplus\left(m^{\prime \prime}\right)$.

Let $J$ be an isomorphism of $\left(m^{\prime \prime}\right)$ onto $\left(m^{\prime}\right) \oplus\left(m^{\prime \prime}\right)$. Define $M$ on $Y$ to $C(H)$ by $M\left(z+x^{\prime \prime}\right)=z+J x^{\prime \prime} 1$ for every $z$ in $Z$ and $x^{\prime \prime}$ in $\left(m^{\prime \prime}\right)$. Then $M$ is an isomorphism of $Y$ with $C(H)$ (cf. [2, p. 391]).

We have shown that $Y$ is either finite dimensional or isomorphic to $C(H)$. To complete the proof, we need only show that $B$ and $Y$ are isomorphic.

Since both $B$ and $Y$ complement $\left(m^{\prime}\right)$ in $C(H), C(H)=B \oplus\left(m^{\prime}\right)$ $=Y \oplus\left(m^{\prime}\right)$. Let $P=I-T$ where $I$ is the identity transformation of $C(H)$ onto $C(H)$ and $T$ is the projection defined above. Since $P x^{\prime}=0$ for $x^{\prime}$ in $\left(m^{\prime}\right), P B=Y$. If $P b=0$ for $b$ in $B$, then $b$ is also in $\left(m^{\prime}\right)$, and it follows that $b=0$. Hence $P$ is an isomorphism of $B$ with $Y$, which completes the proof.

\section{REFERENCES}

1. M. M. Day, Normed linear spaces, Academic Press, New York and Springer, Berlin, 1962.

2. D. W. Dean, Projections in certain continuous function spaces, Canad. J. Math. 14 (1962), 385-401.

3. L. Gillman and M. Jerison, Rings of continuous functions, Van Nostrand, Princeton, N. J., 1960.

4. D. B. Goodner, Projections in normed linear spaces, Trans. Amer. Math. Soc. 69 (1950), 89-108.

5. - The closed convex hull of certain extreme points, Proc. Amer. Math. Soc. 15 (1964), 256-258.

6. R. C. James, Projections in the space $(m)$, Proc. Amer. Math. Soc. 6 (1955), 899-902.

7. A. Pelczynski, Projections in certain Banach spaces, Studia Math. 19 (1960), 209-228.

8. R. S. Phillips, On linear transformations, Trans. Amer, Math. Soc. 48 (1940), 516-541.

Florida State University 